play the major part in the formation of metaphase bivalents, as is evident by the ring-like configurations at this stage. The kinetochores do not appear to show any repulsion whatever, at least in some of the bivalents. In fact the homologues of each bivalent appear to be held together, at the metaphase, by the residual affinity or the terminal attraction even in the absence of any chiasma.

A detailed account of the structure and behaviour of the chromosomes in $P$. americana will shortly be published elsewhere.

Department of Zoology,

G. P. Sharma

Ram Parshad

Prem Sehgal

University of the Panjab,

Hoshiarpur.

July 2.

${ }^{1}$ Morse, Max, Arch. Zellforsch., 3, 483 (1909).

2 Suomalainen, E., Ann. Acad. Sci. Fenn., 4, 1 (1946).

3 Hughes-Schrader, S., "Adv. Genet.", 2, 127 (1948).

- Federley, H., Soc. Scient. Fennicae, Comm. Biol., 9, 1 (1945).

s Hughes-Schrader, S., J. Morph., 73, 111 (1943).

'Darlington, C. D., "Recent Advances in Cytology" (J. and A. Churchill, Ltd., London, 1932).

\section{Crossing-over in Drosophila willistoni}

IN the course of analysing the frequency of chromosomal inversions in 292 adult females of Drosophila willistoni, we have recorded for the first time evidence of crossing-over between two nonoverlapping inversions in the same chromosome arm of this species. These findings are of some general interest since, as Dobzhansky and Epling ${ }^{1}$ have said: "The biological function of inversions in natural populations of Drosophila and their principal role in the evolution of these insects might be conceived to be the suppression of crossing over between gene complexes which have reached an adaptive equilibrium. Crossing over would destroy these complexes and would result in gene combinations of different adaptive values, but would be prevented by the binding effect of the inversions".

In D. pseudo-obscura Dobzhansky and Epling found a strong suppression of crossing-over in the third chromosome due to inversion heterozygosis. Such suppression of crossing-over is adaptive, for the reasons Dobzhansky and Epling give. At the same time suppression of crossing-over reduces one source of genetic plasticity. However, there is gain in plasticity in other ways since crossing-over appears to be intensified on those chromosomes without inversions in individuals heterozygous for inversions on a given chromosome. This is true of $D$. pseudoobscura as well as of other species ${ }^{2}$. In $D$. willistoni the inversions occur on all chromosomes. Suppression of crossing-over on all chromosomes would then cause a great reduction in genetic plasticity. But this does not seem to occur, as the following results indicate.

We have examined the frequency of inversion heterozygotes of six offspring of each of 292 crosses of wild virgin females with males of a strain homozygous for its gene arrangements. Table 1 shows the minimal frequency with which crossing-over occurred between inversions $B$ and $J$ on the third chromosome and between inversions $E$ and $F$ on the left arm of the second chromosome. These are minimal frequencies since not all crossing-over will be recorded in this way. There were insufficient numbers of pairs of heterozygous inversions on the Table 1. Minimal estimates for the frequency (per cent) of crossing-over
between inversions $E$ and $F$ in the left arm of the second chromosome and between inversions $B$ and $J$ in the third chromosome of Drosophila and between inversions $B$ and $J$ in the third chromosome of $D$ ros
willistoni. Flies were bred from three different fruits

\begin{tabular}{|c|c|c|c|}
\hline \multirow{2}{*}{ Fruit } & \multicolumn{2}{|c|}{$\begin{array}{l}\text { Chromosome and } \\
\text { inversion }\end{array}$} & \multirow{2}{*}{ No. of flies } \\
\hline & $\begin{array}{l}I I L \\
\text { and } F\end{array}$ & $\begin{array}{l}I I I \\
\text { and } J\end{array}$ & \\
\hline \multirow{2}{*}{$\begin{array}{l}\text { Philodendron sp. } \\
\text { Psidium arafa } \\
\text { Arecastrum } \\
\quad \text { romanzofianum }\end{array}$} & $\begin{array}{l}23 \\
20\end{array}$ & $\begin{array}{r}9 \\
11\end{array}$ & $\begin{array}{l}100 \\
100\end{array}$ \\
\hline & 16 & 15 & 92 \\
\hline Mean & $19 \cdot 7$ & $11 \cdot 7$ & \\
\hline
\end{tabular}

other chromosomes for us to obtain estimates for these chromosomes. The flies were bred from three different wild fruits. There are no significant differences between the fruits, so the samples may be regarded as replicates. For chromosome $I I L$ the minimal mean frequency of crossing-over between inversions $E$ and $F$ was $19 \cdot 7$. This is remarkably high as these two inversions are quite close to one another on the chromosome. For chromosome III the mean frequency of crossing-over between inversions $B$ and $J$ was $11 \cdot 7$. This is less remarkable as the distance between these two inversions is quite large, $B$ being at the base of the chromosomes and $J$ near the tip.

These high frequencies of crossing-over in $D$. willistoni are quite in conformity with the principle formulated by Dobzhansky and Epling ${ }^{1}$ to which we have already referred. It is what might be ex. pected to happen in a species in which inversions are spread through all the chromosomes. The inversions maintain the integrity of blocks of genes within the inversions but do not inhibit recombination as between inversions.

\section{Bruno Battaglia}

\section{C. BIRCH}

Institute of Zoology, University of Padua;

Department of Zoology,

University of Sydney;

Department of General Biology,

University "of São Paulo, Brazil.

${ }^{1}$ Dobzhansky, Th., and EEpling, Carl, Proc. U.S. Nat. Acad. Sci., 34, 137 (1948).

${ }^{2}$ Da Cunha, A. B., Adv. in Gen., 7, 93 (1955).

\section{Chromosomes of the Cultivated Mushroom}

Since the original work by Maire ${ }^{1}$, the attention of a number of cytologists ${ }^{2-4}$ has been attracted to the study of nuclear behaviour and basidiospore formation in the two-spored cultivated mushroom, Agaricus campestris Fr. var. bisporus ${ }^{5}$. Sass ${ }^{2}$ first published a full account of meiosis in the basidium, and although in one of his diagrams eight bivalents are clearly figured he reported a diploid chromosome number of approximately eight, a determination which was later confirmed ${ }^{4}$. In the interim, however, Colson ${ }^{3}$, after a most comprehensive study, reported a chromosome number of $n=9$.

The results of an investigation using a variety of mushroom grown on a commercial scale in Great Britain have proved of considerable interest, and a chromosome number differing from the two earlier recorded numbers has been found. In this material, 